PESQUIMAT Revista de la Fac. CC. MM. de la

Universidad Nacional Mayor de San Marcos

Vol. IX N², pp 31 - 49, LIMA - PERÚ. Diciembre 2006

\title{
Espectro de Fučik para el problema de valor frontera Sturm-Liouville
}

\author{
Santiago César Rojas Romero ${ }^{1}$ \\ srojasr@unmsm.edu.pe
}

\begin{abstract}
Resumen
En este trabajo consideramos la ecuación de Fučik en $\Omega=\langle 0, \pi>$ con condiciones de frontera Sturm-Liouville. Obtenemos su espectro de Fučik, así como la descripción completa y las propiedades de las curvas contenidas en él.

Palabras Clave: Ecuación de Fučik, Condiciones de frontera tipo Sturm-Liouville, Espectro de Fučik.
\end{abstract}

\begin{abstract}
In this article, we consider the Fučik equation in $\Omega=<0, \pi>$ with Sturm-Liouville boundary conditions. We obtain its Fučik spectrum also the complete description and properties of the curves on it.
\end{abstract}

Keywords: Fučik equation, Sturm-Liouville boundary conditions, Fučik Spectrum.

\section{Introducción}

La ecuación diferencial

$$
w^{\prime \prime}=-\mu^{2} w^{+}+\lambda^{2} w^{-},
$$

donde $w^{+}=\operatorname{máx}\{w, 0\}$ y $w^{-}=$máx $\{-w, 0\}$, es llamada la ecuación de Fučik. Consideramos el siguiente problema de valor frontera Sturm-Liouville

$$
\left\{\begin{array}{l}
w^{\prime \prime}=-\mu^{2} w^{+}+\lambda^{2} w^{-} \\
w(0) \cos \alpha-w^{\prime}(0) \operatorname{sen} \alpha=0 \\
w(\pi) \cos \beta-w^{\prime}(\pi) \operatorname{sen} \beta=0
\end{array}\right.
$$

donde

$$
0 \leq \alpha \leq \frac{\pi}{2} \leq \beta \leq \pi
$$

\footnotetext{
${ }^{1}$ UNMSM, Facultad de Ciencias Matemáticas, Lima - Perú.
} 
En el presente trabajo hallaremos el Espectro de Fučik de este problema, es decir, hallaremos el conjunto de pares $(\lambda, \mu)$ para los cuales el problema (1.1) tiene soluciones no triviales.

En Fučik [2] y Rojas [3] se hace un estudio del espectro de Fučik para el caso $\alpha=0$ y $\beta=\pi$, es decir para el problema tipo Dirichlet:

$$
\left\{\begin{array}{l}
w^{\prime \prime}=-\mu^{2} w^{+}+\lambda^{2} w^{-} \\
w(0)=w(\pi)=0
\end{array}\right.
$$

obteniendo que éste consta de curvas separadas. Más exactamente, se prueba que $(\lambda, \mu)$ está en el espectro de Fučik de (1.2) si y sólo si se cumple una de las siguientes condiciones:

- $\mu=1, \lambda$ arbitrario ,

- $\mu$ arbitrario , $\lambda=1$,

- $\mu>1, \lambda>1, \quad \frac{k}{\mu}+\frac{k}{\lambda}=1, \quad k \in \mathbb{N}$,

- $\mu>1, \lambda>1, \quad \frac{k+1}{\mu}+\frac{k}{\lambda}=1, \quad k \in \mathbb{N}$,

- $\mu>1, \lambda>1, \quad \frac{k}{\mu}+\frac{k+1}{\lambda}=1, \quad k \in \mathbb{N}$.

Estas curvas también pueden escribirse como:

$$
\begin{aligned}
F_{0}^{+}: & \mu=1, \\
F_{0}^{-}: & \lambda=1, \\
F_{2 k-1}^{ \pm}: & \frac{k}{\mu}+\frac{k}{\lambda}=1, \\
F_{2 k}^{+}: & \frac{k+1}{\mu}+\frac{k}{\lambda}=1, \\
F_{2 k}^{-}: & \frac{k}{\mu}+\frac{k+1}{\lambda}=1 \quad, \quad \forall k \in \mathbb{N},
\end{aligned}
$$

donde el subíndice indica el número de ceros de las respectivas soluciones no triviales $w$ de (1.2) en el intervalo $\left\langle 0, \pi>\right.$ y el signo + ó - indica si $w^{\prime}(0)>0$ ó $w^{\prime}(0)<0$, respectivamente.

Nuestro estudio para el caso general

$$
0 \leq \alpha \leq \frac{\pi}{2} \leq \beta \leq \pi
$$

está basado en la reducción del problema (1.1) a otro de primer orden introduciendo coordenadas polares.

La parte restante del trabajo está organizada como sigue: en la sección 2 enunciamos y probamos nuestros principales resultados. En las secciones 3 y 4 obtenemos las expresiones analíticas y las propiedades de las diferentes ramas del espectro de Fučik del problema (1.1). Finalmente, en la sección 5 presentamos dos casos específicos del problema en estudio. 


\section{Coordenadas Polares y Principales Resultados}

Tenemos como centro de nuestro estudio la ecuación diferencial

$$
w^{\prime \prime}=-\mu^{2} w^{+}+\lambda^{2} w^{-} .
$$

Introducimos las coordenadas polares

$$
\begin{gathered}
w=\rho \operatorname{sen} \varphi \\
w^{\prime}=\rho \cos \varphi
\end{gathered}
$$

donde $\rho(t)$ es la distancia de un punto $\left(w(t), w^{\prime}(t)\right)$ al origen y $\varphi(t)$ es el ángulo entre el vector $\left(w(t), w^{\prime}(t)\right)$ y el eje vertical.

Así, la función $f(w)=-\mu^{2} w^{+}+\lambda^{2} w^{-}$en coordenadas polares se escribe como

$$
f(\rho, \varphi)= \begin{cases}-\mu^{2} \rho \operatorname{sen} \varphi & , \quad \operatorname{sen} \varphi \geq 0 \\ -\lambda^{2} \rho \operatorname{sen} \varphi & , \quad \operatorname{sen} \varphi<0\end{cases}
$$

y tenemos la siguiente proposición

Proposición 2.1 Para las soluciones de la ecuación (2.1) con condiciones iniciales $\varphi(0)=$ $\varphi_{0} \quad y \quad \rho(0)=\rho_{0}$, el ángulo $\varphi(t)$ cambia al valor $\varphi(T)=\varphi$ en el tiempo $T$, para cualquier $\rho_{0}>0$.

Prueba.- Derivando (2.2) respecto a t, obtenemos

$$
w^{\prime}=\rho^{\prime} \operatorname{sen} \varphi+\rho \varphi^{\prime} \cos \varphi
$$

y usando (2.3) tenemos

$$
\rho^{\prime} \operatorname{sen} \varphi+\rho \varphi^{\prime} \cos \varphi=\rho \cos \varphi
$$

Ahora derivamos (2.3) respecto a t y obtenemos

$$
w^{\prime \prime}=\rho^{\prime} \cos \varphi-\rho \varphi^{\prime} \operatorname{sen} \varphi
$$

Usando (2.1) llegamos a

$$
\rho^{\prime} \cos \varphi-\rho \varphi^{\prime} \operatorname{sen} \varphi=f(\rho, \varphi)
$$

Así , en lugar de la ecuación (2.1) llegamos al sistema formado por las ecuaciones (2.5) y (2.6)

$$
\left\{\begin{array}{l}
\rho^{\prime} \operatorname{sen} \varphi+\rho \varphi^{\prime} \cos \varphi=\rho \cos \varphi \\
\rho^{\prime} \cos \varphi-\rho \varphi^{\prime} \operatorname{sen} \varphi=f(\rho, \varphi)
\end{array}\right.
$$

El cual nos conduce al sistema

$$
\left\{\begin{array}{l}
\rho^{\prime}=\rho \operatorname{sen} \varphi \cos \varphi+f(\rho, \varphi) \cos \varphi \\
\varphi^{\prime}=-\frac{1}{\rho} f(\rho, \varphi) \operatorname{sen} \varphi+\cos ^{2} \varphi
\end{array}\right.
$$


Y usando (2.4), tenemos

$$
\varphi^{\prime}=F(\varphi)= \begin{cases}\mu^{2} \operatorname{sen}^{2} \varphi+\cos ^{2} \varphi & , \quad \operatorname{sen} \varphi \geq 0 \\ \lambda^{2} \operatorname{sen}^{2} \varphi+\cos ^{2} \varphi & , \quad \operatorname{sen} \varphi<0\end{cases}
$$

Como puede verse en (2.8), la función $\varphi(t)$ es creciente pues $\varphi^{\prime}>0$, y la derivada de $\varphi(t)$ es independiente de $\rho(t)$.

De esta manera, nuestro problema en estudio se convierte en

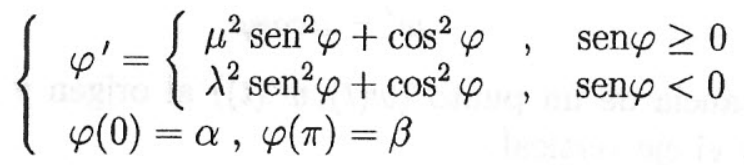

donde

$$
0 \leq \alpha \leq \frac{\pi}{2} \leq \beta \leq \pi
$$

La interpretación geométrica de este problema es : hallar la curva integral que empieza en la recta $\varphi(0)=\alpha \quad$ y termina en $\varphi(\pi)=\beta$.

A continuación presentamos los resultados que nos permiten resolver este problema .

Proposición 2.2 La solución del problema

$$
\left\{\begin{array}{l}
\varphi^{\prime}=k^{2} \operatorname{sen}^{2} \varphi+\cos ^{2} \varphi, k>0 \\
\varphi\left(t_{0}\right)=\varphi_{0},
\end{array}\right.
$$

siendo $0 \leq \varphi_{0} \leq \varphi \leq \frac{\pi}{2}$, es dada por

$$
\frac{1}{k} \arctan (k \tan \varphi)-\frac{1}{k} \arctan \left(k \tan \varphi_{0}\right)=t-t_{0} .
$$

Prueba.- De la ecuación, tenemos

$$
\frac{d \varphi}{k^{2} \operatorname{sen}^{2} \varphi+\cos ^{2} \varphi}=d t
$$

Ahora, integramos y llegamos a

$$
\int_{t_{0}}^{t} d t=\int_{\varphi_{0}}^{\varphi} \frac{d \varphi}{k^{2} \operatorname{sen}^{2} \varphi+\cos ^{2} \varphi}=\int_{\varphi_{0}}^{\varphi} \frac{\sec ^{2} \varphi d \varphi}{k^{2} \tan ^{2} \varphi+1}=\frac{1}{k} \int_{\varphi_{0}}^{\varphi} \frac{d(k \tan \varphi)}{(k \tan \varphi)^{2}+1}
$$

es decir

$$
t-t_{0}=\left.\frac{1}{k} \arctan (k \tan \varphi)\right|_{\varphi_{0}} ^{\varphi}=\frac{1}{k} \arctan (k \tan \varphi)-\frac{1}{k} \arctan \left(k \tan \varphi_{0}\right)
$$


Proposición 2.3 La solución del problema

$$
\left\{\begin{array}{l}
\varphi^{\prime}=k^{2} \operatorname{sen}^{2} \varphi+\cos ^{2} \varphi, k>0 \\
\varphi\left(t_{0}\right)=\varphi_{0}
\end{array}\right.
$$

siendo $0 \leq \varphi_{0}<\frac{\pi}{2}<\varphi \leq \pi$, es dada por

$$
\frac{1}{k} \arctan (k \tan \varphi)+\frac{\pi}{k}-\frac{1}{k} \arctan \left(k \tan \varphi_{0}\right)=t-t_{0}
$$

Prueba.- Por hipótesis $\frac{\pi}{2} \in\left\langle\varphi_{0}, \varphi\right\rangle$. Entonces la integral de la derecha en

$$
\int_{t_{0}}^{t} d t=\int_{\varphi_{0}}^{\varphi} \frac{d \varphi}{k^{2} \operatorname{sen}^{2} \varphi+\cos ^{2} \varphi}
$$

es igual a la suma de dos integrales:

$$
\begin{aligned}
t-t_{0}= & \frac{1}{k} \int_{\varphi_{0}}^{\varphi} \frac{d(k \tan \varphi)}{(k \tan \varphi)^{2}+1} \\
= & \frac{1}{k}\left\{\int_{\varphi_{0}}^{\frac{\pi}{2}} \frac{d(k \tan \varphi)}{(k \tan \varphi)^{2}+1}+\int_{\frac{\pi}{2}}^{\varphi} \frac{d(k \tan \varphi)}{(k \tan \varphi)^{2}+1}\right\} \\
= & \frac{1}{k}\left\{\lim _{\epsilon_{1} \rightarrow 0} \int_{\varphi_{0}}^{\frac{\pi}{2}-\epsilon_{1}} \frac{d(k \tan \varphi)}{(k \tan \varphi)^{2}+1}+\lim _{\epsilon_{2} \rightarrow 0} \int_{\frac{\pi}{2}+\epsilon_{2}}^{\varphi} \frac{d(k \tan \varphi)}{(k \tan \varphi)^{2}+1}\right\} \\
= & \frac{1}{k}\left\{\lim _{\epsilon_{1} \rightarrow 0}\left[\arctan \left(k \tan \left(\frac{\pi}{2}-\epsilon_{1}\right)\right)-\arctan \left(k \tan \varphi_{0}\right)\right]+\right. \\
& \left.\quad+\lim _{\epsilon_{2} \rightarrow 0}\left[\arctan (k \tan \varphi)-\arctan \left(k \tan \left(\frac{\pi}{2}+\epsilon_{2}\right)\right)\right]\right\} \\
= & \frac{1}{k}\left\{\frac{\pi}{2}-\arctan \left(k \tan \varphi_{0}\right)+\arctan (k \tan \varphi)+\frac{\pi}{2}\right\} \\
= & \frac{1}{k} \arctan (k \tan \varphi)+\frac{\pi}{k}-\frac{1}{k} \arctan \left(k \tan \varphi_{0}\right) \cdot \square
\end{aligned}
$$

Proposición 2.4 Sea el problema

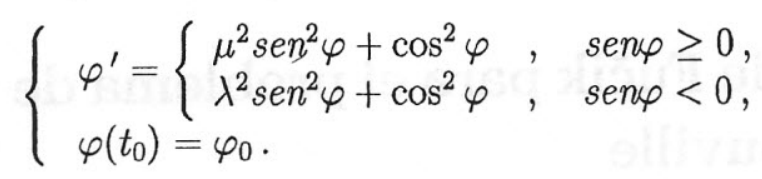

Si $2 n \pi \leq \varphi_{0} \leq \varphi \leq \frac{\pi}{2}+2 n \pi \quad$ o $\frac{\pi}{2}+2 n \pi \leq \varphi_{0} \leq \varphi \leq \pi+2 n \pi, n=0,1,2, \ldots$, entonces la solución satisface

$$
\frac{1}{\mu} \arctan (\mu \tan \varphi)-\frac{1}{\mu} \arctan \left(\mu \tan \varphi_{0}\right)=t-t_{0} .
$$

Si $2 n \pi \leq \varphi_{0}<\frac{\pi}{2}+2 n \pi<\varphi \leq \pi+2 n \pi, n=0,1,2, \ldots$, entonces la solución satisface

$$
\frac{1}{\mu} \arctan (\mu \tan \varphi)+\frac{\pi}{\mu}-\frac{1}{\mu} \arctan \left(\mu \tan \varphi_{0}\right)=t-t_{0}
$$


Prueba.- Por hipótesis, $2 n \pi \leq \varphi_{0} \leq \varphi \leq \pi+2 n \pi$ y por ello $\operatorname{sen} \varphi \geq 0$. Luego, en este intervalo de valores de $\varphi$, el problema se reduce a

$$
\left\{\begin{array}{l}
\varphi^{\prime}=\mu^{2} \operatorname{sen}^{2} \varphi+\cos ^{2} \varphi \\
\varphi\left(t_{0}\right)=\varphi_{0}
\end{array}\right.
$$

Usando las proposiciones 2.2 y 2.3 obtenemos el resultado

Proposición 2.5 Sea el problema

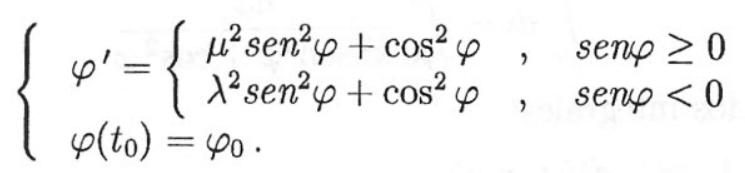

Si $\pi+2 n \pi \leq \varphi_{0} \leq \varphi \leq \frac{3 \pi}{2}+2 n \pi \quad o \quad \frac{3 \pi}{2}+2 n \pi \leq \varphi_{0} \leq \varphi \leq 2 \pi+2 n \pi, n=0,1,2, \ldots$, entonces la solución satisface

$$
\frac{1}{\lambda} \arctan (\lambda \tan \varphi)-\frac{1}{\lambda} \arctan \left(\lambda \tan \varphi_{0}\right)=t-t_{0}
$$

Si $\pi+2 n \pi \leq \varphi_{0} \leq \frac{3 \pi}{2}+2 n \pi \leq \varphi \leq 2 \pi+2 n \pi, n=0,1,2, \ldots$, entonces la solución satisface

$$
\frac{1}{\lambda} \arctan (\lambda \tan \varphi)+\frac{\pi}{\lambda}-\frac{1}{\lambda} \arctan \left(\lambda \tan \varphi_{0}\right)=t-t_{0}
$$

Prueba.- De la hipótesis, $\pi+2 n \pi \leq \varphi_{0} \leq \varphi \leq 2 \pi+2 n \pi$ y de ahí que $\operatorname{sen} \varphi \leq 0$. Luego, en este intervalo de valores de $\varphi$, el problema se reduce a

$$
\left\{\begin{array}{l}
\varphi^{\prime}=\lambda^{2} \operatorname{sen}^{2} \varphi+\cos ^{2} \varphi \\
\varphi\left(t_{0}\right)=\varphi_{0}
\end{array}\right.
$$

Nuevamente, usando las proposiciones 2.2 y 2.3 obtenemos el resultado .

\section{Espectro de Fučik para el problema de valor frontera Sturm-Liouville}

En esta sección obtendremos el Espectro de Fučik para el problema

$$
\left\{\begin{array}{l}
w^{\prime \prime}=-\mu^{2} w^{+}+\lambda^{2} w^{-} \\
w(0) \cos \alpha-w^{\prime}(0) \operatorname{sen} \alpha=0, \\
w(\pi) \cos \beta-w^{\prime}(\pi) \operatorname{sen} \beta=0, \quad 0 \leq \alpha \leq \frac{\pi}{2} \leq \beta \leq \pi
\end{array}\right.
$$

Denotamos con $F_{k}^{+}$y $F_{k}^{-}, k=0,1,2, \ldots$, las diferentes ramas del espectro, donde el subíndice indica el número exacto de ceros que tiene las respectivas soluciones no triviales $w(t)$ en el 
intervalo $<0, \pi>$ y el signo indica si $w^{\prime}(0)>0$ ó $w^{\prime}(0)<0$.

Hallaremos el espectro de (3.1) resolviendo la ecuación

$$
\varphi^{\prime}=\left\{\begin{array}{l}
\mu^{2} \operatorname{sen}^{2} \varphi+\cos ^{2} \varphi, \text { si } \operatorname{sen} \varphi \geq 0 \\
\lambda^{2} \operatorname{sen}^{2} \varphi+\cos ^{2} \varphi, \text { si } \operatorname{sen} \varphi<0
\end{array}\right.
$$

con las condiciones de frontera $\varphi(0)=\alpha, \varphi(\pi)=\beta$.

En primer lugar, obtendremos las ecuaciones de las ramas $F_{0}^{+}, F_{0}^{-}, F_{1}^{+}, F_{1}^{-}, F_{2}^{+}$y $F_{2}^{-}$. Luego, generalizamos el estudio obteniendo fórmulas para las ramas $F_{k}^{+}$y $F_{k}^{-}, \forall k \in \mathbb{N}$.

\subsection{La rama $F_{0}^{+}$}

Esta rama del espectro de (3.1) está formada por todos los puntos $(\lambda, \mu)$ que dan lugar a soluciones $w(t)$ del problema (3.1) tales que $w(t)>0, \forall t \in\langle 0, \pi\rangle$.

Por las condiciones de frontera, la recta asociada a estas soluciones gira en el plano de fase un ángulo igual a $\beta-\alpha$ en el tiempo $T=\pi$, y para cualquier $t$ en este intervalo de tiempo tenemos que $\operatorname{sen} \varphi \geq 0$.

Entonces, el problema (3.2) con las condiciones de frontera se reduce al problema

$$
\left\{\begin{array}{l}
\varphi^{\prime}=\mu^{2} \operatorname{sen}^{2} \varphi+\cos ^{2} \varphi \\
\varphi(0)=\alpha \\
\varphi(\pi)=\beta
\end{array}\right.
$$

y usando la proposición 2.4 , tenemos que

$$
\frac{1}{\mu} \arctan (\mu \tan \beta)+\frac{\pi}{\mu}-\frac{1}{\mu} \arctan (\mu \tan \alpha)=\pi .
$$

Así, la rama $F_{0}^{+}$consiste en todos los puntos $(\lambda, \mu)$ que resuelven la ecuación (3.3).

\subsection{La rama $F_{0}^{-}$}

Esta rama del espectro está formada por todos los puntos $(\lambda, \mu)$ que dan lugar a soluciones $w(t)$ del problema (3.1) que satisfacen $w(t)<0, \forall t \in<0, \pi>$.

Para estas soluciones, la recta asociada a ellas en el plano de fase gira un ángulo igual a $\beta-\alpha$ en el tiempo $T=\pi$, y para todo $t$ en este intervalo de tiempo se tiene que $\operatorname{sen} \varphi \leq 0$. Entonces, el problema (3.2) con las condiciones de frontera se reduce al problema

$$
\left\{\begin{array}{l}
\varphi^{\prime}=\lambda^{2} \operatorname{sen}^{2} \varphi+\cos ^{2} \varphi \\
\varphi(0)=\alpha+\pi \\
\varphi(\pi)=\beta+\pi
\end{array}\right.
$$

De aquí, usando la proposición 2.5, tenemos que

$$
\frac{1}{\lambda} \arctan (\lambda \tan (\beta+\pi))+\frac{\pi}{\lambda}-\frac{1}{\lambda} \arctan (\lambda \tan (\alpha+\pi))=\pi,
$$


o lo que es igual a

$$
\frac{1}{\lambda} \arctan (\lambda \tan \beta)+\frac{\pi}{\lambda}-\frac{1}{\lambda} \arctan (\lambda \tan \alpha)=\pi .
$$

Así, la rama $F_{0}^{-}$consiste en todos los puntos $(\lambda, \mu)$ que resuelven la ecuación (3.4).

\subsection{La rama $F_{1}^{+}$}

Esta rama está formada por todos los puntos $(\lambda, \mu)$ que dan lugar a soluciones $w(t)$ del problema (3.1), las cuales tienen exactamente un cero en el intervalo $<0, \pi>$ y satisfacen $w^{\prime}(0)>0$.

En este caso, la recta asociada a tales soluciones gira en el plano de fase un ángulo igual a $\pi+\beta-\alpha$ en el tiempo $T=\pi$. El intervalo $[0, \pi]$ es descompuesto en dos intervalos $J_{T_{1}}:=\left[0, T_{1}\right]$ y $J_{T_{2}}:=\left[T_{1}, \pi\right]$, donde $T_{2}:=\pi-T_{1}$. La recta en el plano de fase gira en el tiempo $T_{1}$ desde el ángulo $\alpha$ hasta $\pi$, y en consecuencia $\operatorname{sen} \varphi \geq 0$. Para el resto del tiempo $T_{2}$, la recta en el plano de fase gira desde el ángulo $\pi$ hasta $\pi+\beta$, y para los respectivos valores de $t$ se tiene que $\operatorname{sen} \varphi \leq 0$.

En el intervalo $J_{T_{1}}$ el problema (3.2) se reduce al problema*

$$
\left\{\begin{array}{l}
\varphi^{\prime}=\mu^{2} \operatorname{sen}^{2} \varphi+\cos ^{2} \varphi \\
\varphi(0)=\alpha \\
\varphi\left(T_{1}\right)=\pi
\end{array}\right.
$$

y en el intervalo $J_{T_{2}}$ se reduce a

$$
\left\{\begin{array}{l}
\varphi^{\prime}=\lambda^{2} \operatorname{sen}^{2} \varphi+\cos ^{2} \varphi \\
\varphi\left(T_{1}\right)=\pi \\
\varphi(\pi)=\beta+\pi
\end{array}\right.
$$

Usando las proposiciones 2.4 y 2.5 , obtenemos que

$$
T_{1}=\frac{1}{\mu} \arctan (\mu \tan \pi)+\frac{\pi}{\mu}-\frac{1}{\mu} \arctan (\mu \tan \alpha)
$$

0

$$
T_{1}=\frac{\pi}{\mu}-\frac{1}{\mu} \arctan (\mu \tan \alpha)
$$

y

$$
\pi-T_{1}=\frac{1}{\lambda} \arctan (\lambda \tan (\beta+\pi))+\frac{\pi}{\lambda}-\frac{1}{\lambda} \arctan (\lambda \tan \pi),
$$

o

$$
T_{2}=\frac{1}{\lambda} \arctan (\lambda \tan \beta)+\frac{\pi}{\lambda} .
$$

Introduciendo la notación $\gamma=\pi-\beta$, tenemos

$$
T_{2}=\frac{\pi}{\lambda}-\frac{1}{\lambda} \arctan (\lambda \tan \gamma)
$$


y como $T_{1}+T_{2}=\pi$, llegamos a la relación

$$
\left[\frac{\pi}{\mu}-\frac{1}{\mu} \arctan (\mu \tan \alpha)\right]+\left[\frac{\pi}{\lambda}-\frac{1}{\lambda} \arctan (\lambda \tan \gamma)\right]=\pi
$$

De este modo, la rama $F_{1}^{+}$consiste en todos los puntos $(\lambda, \mu)$ que satisfacen la ecuación (3.5).

\subsection{La rama $F_{1}^{-}$}

Esta rama la forman todos los puntos $(\lambda, \mu)$ que dan lugar a soluciones $w(t)$ del problema (3.1), las cuales tienen exactamente un cero en el intervalo $<0, \pi>$, y satisfacen $w^{\prime}(0)<0$. La respectiva recta en el plano de fase gira un ángulo igual a $\pi+\beta-\alpha$ en el tiempo $T=\pi$. El intervalo $[0, \pi]$ se descompone en los intervalos $J_{T_{1}}:=\left[0, T_{1}\right]$ y $J_{T_{2}}:=\left[T_{1}, \pi\right]$, donde $T_{2}:=\pi-T_{1}$. En el tiempo $T_{1}$, la respectiva recta en el plano de fase gira desde el ángulo $\pi+\alpha$ hasta $2 \pi$, y para $t \in J_{T_{1}}$ se tiene que $\operatorname{sen} \varphi \leq 0$. En el tiempo restante $T_{2}$ la recta gira desde el ángulo $2 \pi$ a $2 \pi+\beta$, y para $t \in J_{T_{2}}$ tenemos que $\operatorname{sen} \varphi \geq 0$.

En el intervalo $J_{T_{1}}$, el problema (3.2) se reduce a la ecuación

$$
\left\{\begin{array}{l}
\varphi^{\prime}=\lambda^{2} \operatorname{sen}^{2} \varphi+\cos ^{2} \varphi \\
\varphi(0)=\pi+\alpha \\
\varphi\left(T_{1}\right)=2 \pi
\end{array}\right.
$$

De la cual, usando la proposición 2.3 obtenemos que

$$
\frac{1}{\lambda} \arctan (\lambda \tan 2 \pi)+\frac{\pi}{\lambda}-\frac{1}{\lambda} \arctan (\lambda \tan (\pi+\alpha))=T_{1}
$$

o

$$
\frac{\pi}{\lambda}-\frac{1}{\lambda} \arctan (\lambda \tan \alpha)=T_{1}
$$

En el intervalo $J_{T_{2}}$, el problema (3.2) se reduce a

$$
\left\{\begin{array}{l}
\varphi^{\prime}=\mu^{2} \operatorname{sen}^{2} \varphi+\cos ^{2} \varphi \\
\varphi\left(T_{1}\right)=2 \pi \\
\varphi(\pi)=2 \pi+\beta
\end{array}\right.
$$

Usando la proposición 2.4, obtenemos que

o

$$
\pi-T_{1}=\frac{1}{\mu} \arctan \mu \tan (2 \pi+\beta)+\frac{\pi}{\mu}-\frac{1}{\mu} \arctan (\mu \tan 2 \pi),
$$

$$
T_{2}=\frac{\pi}{\mu}-\frac{1}{\mu} \arctan (\mu \tan \gamma)
$$

donde $\gamma=\pi-\beta$. Y como $T_{1}+T_{2}=\pi$, tenemos la siguiente relación

$$
\left[\frac{\pi}{\lambda}-\frac{1}{\lambda} \arctan (\lambda \tan \alpha)\right]+\left[\frac{\pi}{\mu}-\frac{1}{\mu} \arctan (\mu \tan \gamma)\right]=\pi .
$$

Así, la rama $F_{1}^{-}$del espectro de (3.1) consiste de todos los puntos $(\lambda, \mu)$ que satisfacen la ecuación (3.6). 


\subsection{La rama $F_{2}^{+}$}

Esta rama está formada por todos los puntos $(\lambda, \mu)$ que dan lugar a soluciones $w(t)$ del problema (3.1), las cuales tienen exactamente dos ceros en el intervalo $<0, \pi>$ y satisfacen $w^{\prime}(0)>0$.

La linea recta asociada a tales soluciones, gira en el plano de fase un ángulo igual a $2 \pi+\beta-\alpha$ en el tiempo $T=\pi$. El intervalo $[0, \pi]$ se descompone en tres intervalos $J_{T_{1}}:=\left[0, T_{1}\right]$, $J_{T_{2}}:=\left[T_{1}, T_{1}+T_{2}\right]$ y $J_{T_{3}}:=\left[\pi-T_{3}, \pi\right]$, donde $T_{3}:=\pi-T_{1}-T_{2}$. En el tiempo $T_{1}$ la recta en el plano de fase gira desde el ángulo $\alpha$ a $\pi$, y por tanto $\operatorname{sen} \varphi \geq 0$; en el tiempo $T_{2}$ gira desde el ángulo $\pi$ a $2 \pi$, y para los respectivos valores de $t, \operatorname{sen} \varphi \leq 0$; en el tiempo $T_{3}$ restante la recta gira desde el ángulo $2 \pi$ a $2 \pi+\beta, y \operatorname{sen} \varphi \geq 0$.

En la subsección 3.3 calculamos el tiempo necesario para la rotación desde el ángulo $\alpha$ a $\pi$, esto es,

$$
T_{1}=\frac{\pi}{\mu}-\frac{1}{\mu} \arctan (\mu \tan \alpha) .
$$

Para hallar el tiempo $T_{2}$, necesario para la rotación del ángulo $\pi$ a $2 \pi$, usamos la proposición 2.3

$$
T_{2}=\frac{1}{\lambda} \arctan (\lambda \tan 2 \pi)+\frac{\pi}{\lambda}-\frac{1}{\lambda} \arctan (\lambda \tan \pi)=\frac{\pi}{\lambda} .
$$

El tiempo necesario para la rotación desde el ángulo $2 \pi$, a $2 \pi+\beta$ ya fue calculado en la subsección 3.4, por tanto el tiempo $T_{3}$ es conocido:

$$
T_{3}=\frac{\pi}{\mu}-\frac{1}{\mu} \arctan (\mu \tan \gamma),
$$

donde $\gamma=\pi-\beta$. Ahora, usamos la relación $T_{1}+T_{2}+T_{3}=\pi$ y llegamos a la fórmula

$$
\left[\frac{\pi}{\mu}-\frac{1}{\mu} \arctan (\mu \tan \alpha)\right]+\frac{\pi}{\lambda}+\left[\frac{\pi}{\mu}-\frac{1}{\mu} \arctan (\mu \tan \gamma)\right]=\pi .
$$

Así, la rama $F_{2}^{+}$consiste en todos los puntos $(\lambda, \mu)$ que satisfacen la ecuación (3.7).

\subsection{La rama $F_{2}^{-}$}

Esta rama del espectro la forman los puntos $(\lambda, \mu)$, para los cuales las soluciones $w(t)$ del problema (3.1) tienen exactamente dos ceros en el intervalo $<0, \pi>$ y satisfacen $w^{\prime}(0)<0$. En este caso, la recta en el plano de fase gira un ángulo igual a $2 \pi+\beta-\alpha$ en el tiempo $T=\pi$. El intervalo se descompone en tres subintervalos: $J_{T_{1}}:=\left[0, T_{1}\right], J_{T_{2}}:=\left[T_{1}, T_{1}+T_{2}\right] \mathrm{Y}$ $J_{T_{3}}:=\left[\pi-T_{1}, \pi\right]$, donde $T_{3}:=\pi-T_{1}-T_{2}$. En el tiempo $T_{1}$, la recta en el plano de fase gira desde el ángulo $\pi+\alpha$ a $2 \pi$. Y por tanto, $\operatorname{sen} \varphi \leq 0$; en el tiempo $T_{2}$, la recta gira desde $2 \pi$ a $3 \pi$ y para esos valores de $t$ se tiene $\operatorname{sen} \varphi \geq 0$; en el tiempo $T_{3}$ restante la recta gira desde el ángulo $3 \pi$ a $3 \pi+\beta$, siendo $\operatorname{sen} \varphi \leq 0$ para estos valores de $t$.

Ahora, usamos los resultados previamente obtenidos. En la subsección 3.4 hallamos el tiempo necesario para que la recta en el plano de fase gire desde el ángulo $\pi+\alpha$ a $2 \pi$, esto es

$$
T_{1}=\frac{\pi}{\lambda}-\frac{1}{\lambda} \arctan (\lambda \tan \alpha) \text {. }
$$


Usando la proposición 2.4, obtenemos que el tiempo $T_{2}$ necesario para que la recta gire de $2 \pi$ a $3 \pi$, es igual a

$$
T_{2}=\frac{1}{\mu} \arctan (\mu \tan 3 \pi)+\frac{\pi}{\mu}-\frac{1}{\mu} \arctan (\mu \tan 2 \pi)=\frac{\pi}{\mu} .
$$

Similarmente, el tiempo $T_{3}$ necesario para el giro desde el ángulo $3 \pi$ a $3 \pi+\beta$, se calcula usando la proposición 2.5:

$$
T_{3}=\frac{\pi}{\lambda}-\frac{1}{\lambda} \arctan (\lambda \tan \gamma)
$$

donde $\gamma=\pi-\beta$. Como $T_{1}+T_{2}+T_{3}=\pi$, obtenemos

$$
\left[\frac{\pi}{\lambda}-\frac{1}{\lambda} \arctan (\lambda \tan \alpha)\right]+\frac{\pi}{\mu}+\left[\frac{\pi}{\lambda}-\frac{1}{\lambda} \arctan (\lambda \tan \gamma)\right]=\pi .
$$

De este modo, la rama $F_{2}^{-}$consiste en todos los puntos $(\lambda, \mu)$ que satisfacen la ecuación (3.8).

\subsection{El caso general}

Sea $w(t)$ una solución no trivial del problema (3.1) que tenga exactamente $n$ ceros en el intervalo $\langle 0, \pi\rangle$. Para esta solución, el intervalo $[0, \pi]$ puede ser descompuesto en $n+1$ subintervalos $J_{T_{1}}:=\left[0, T_{1}\right], J_{T_{2}}:=\left[T_{1}, T_{1}+T_{2}\right], J_{T_{3}}:=\left[T_{1}+T_{2}, T_{1}+T_{2}+T_{3}\right], \ldots, J_{T_{n+1}}=\left[\sum_{i=1}^{n} T_{i}, \pi\right]$ de manera que en cualquiera de estos intervalos el signo de $w(t)$ no cambia.

Observación 3.1 1. Si $n=2 k$, w(t) tiene el mismo signo en los intervalos $J_{T_{1}}$ y $J_{T_{n+1}}$.

2. Si $n=2 k-1, w(t)$ tiene signos diferentes en los intervalos $J_{T_{1}}$ y $J_{T_{n+1}}$.

Usamos las proposiciones 2.3, 2.4, 2.5 y la anterior observación para obtener la descripción analítica de las ramas $F_{n}^{+}$y $F_{n}^{-}$. Así llegamos a que la descomposición del intervalo $[0, \pi]$ en subintervalos es tal que

- Para $F_{2 k}^{+}$:

$$
\begin{aligned}
T_{1} & =\frac{\pi}{\mu}-\frac{1}{\mu} \arctan (\mu \tan \alpha), \\
T_{2} & =T_{4}=T_{6}=\ldots=T_{2 k-2}=T_{2 k}=\frac{\pi}{\lambda} \\
T_{3} & =T_{5}=T_{7}=\ldots=T_{2 k-1}=\frac{\pi}{\mu} \\
T_{2 k+1} & =\frac{\pi}{\mu}-\frac{1}{\mu} \arctan (\mu \tan \gamma) .
\end{aligned}
$$

- Para $F_{2 k}^{-}$:

$$
\begin{aligned}
T_{1} & =\frac{\pi}{\lambda}-\frac{1}{\lambda} \arctan (\lambda \tan \alpha), \\
T_{2} & =T_{4}=T_{6}=\ldots=T_{2 k-2}=T_{2 k}=\frac{\pi}{\mu} \\
T_{3} & =T_{5}=T_{7}=\ldots=T_{2 k-1}=\frac{\pi}{\lambda} \\
T_{2 k+1} & =\frac{\pi}{\lambda}-\frac{1}{\lambda} \arctan (\lambda \tan \gamma) .
\end{aligned}
$$


- Para $F_{2 k-1}^{+}$:

$$
\begin{aligned}
T_{1} & =\frac{\pi}{\mu}-\frac{1}{\mu} \arctan (\mu \tan \alpha) \\
T_{2} & =T_{4}=T_{6}=\ldots=T_{2 k-2}=\frac{\pi}{\lambda} \\
T_{3} & =T_{5}=T_{7}=\ldots=T_{2 k-1}=\frac{\pi}{\mu} \\
T_{2 k} & =\frac{\pi}{\lambda}-\frac{1}{\lambda} \arctan (\lambda \tan \gamma) .
\end{aligned}
$$

- Para $F_{2 k-1}^{-}$

$$
\begin{aligned}
T_{1} & =\frac{\pi}{\lambda}-\frac{1}{\lambda} \arctan (\lambda \tan \alpha), \\
T_{2} & =T_{4}=T_{6}=\ldots=T_{2 k-2}=\frac{\pi}{\mu} \\
T_{3} & =T_{5}=T_{7}=\ldots=T_{2 k-1}=\frac{\pi}{\lambda} \\
T_{2 k} & =\frac{\pi}{\mu}-\frac{1}{\mu} \arctan (\mu \tan \gamma) .
\end{aligned}
$$

Como la suma de todos los intervalos $J_{T_{1}}, J_{T_{2}}, J_{T_{3}}, \ldots, J_{T_{n+1}}$ es $\pi$ :

$$
\sum_{i=1}^{n+1} T_{i}=\pi
$$

obtenemos ecuaciones para las ramas del espectro de Fučik del problema (3.1) , las cuales quedan establecidas en la siguiente proposición.

Proposición 3.1 Las ramas del espectro de Fučik para el problema de valor frontera

$$
\left\{\begin{array}{l}
w^{\prime \prime}=-\mu^{2} w^{+}+\lambda^{2} w^{-}, \\
w(0) \cos \alpha-w^{\prime}(0) \operatorname{sen} \alpha=0, \\
w(\pi) \cos \beta-w^{\prime}(\pi) \operatorname{sen} \beta=0, \quad 0 \leq \alpha \leq \frac{\pi}{2} \leq \beta \leq \pi,
\end{array}\right.
$$

son dadas por las ecuaciones:

$$
\begin{aligned}
& F_{0}^{+}: \frac{\pi}{\mu}-\frac{1}{\mu} \arctan (\mu \tan \gamma)-\frac{1}{\mu} \arctan (\mu \tan \alpha)=\pi \\
& F_{0}^{-}: \frac{\pi}{\lambda}-\frac{1}{\lambda} \arctan (\lambda \tan \gamma)-\frac{1}{\lambda} \arctan (\lambda \tan \alpha)=\pi
\end{aligned}
$$

$F_{2 k}^{+}:\left[\frac{\pi}{\mu}-\frac{1}{\mu} \arctan (\mu \tan \alpha)\right]+\frac{(k-1) \pi}{\mu}+\frac{k \pi}{\lambda}+\left[\frac{\pi}{\mu}-\frac{1}{\mu} \arctan (\mu \tan \gamma)\right]=\pi$,

$F_{2 k}^{-}:\left[\frac{\pi}{\lambda}-\frac{1}{\lambda} \arctan (\lambda \tan \alpha)\right]+\frac{k \pi}{\mu}+\frac{(k-1) \pi}{\lambda}+\left[\frac{\pi}{\lambda}-\frac{1}{\lambda} \arctan (\lambda \tan \gamma)\right]=\pi$,

$F_{2 k-1}^{+}:\left[\frac{\pi}{\mu}-\frac{1}{\mu} \arctan (\mu \tan \alpha)\right]+\frac{(k-1) \pi}{\mu}+\frac{(k-1) \pi}{\lambda}+\left[\frac{\pi}{\lambda}-\frac{1}{\lambda} \arctan (\lambda \tan \gamma)\right]=\pi$,

$F_{2 k-1}^{-}:\left[\frac{\pi}{\lambda}-\frac{1}{\lambda} \arctan (\lambda \tan \alpha)\right]+\frac{(k-1) \pi}{\mu}+\frac{(k-1) \pi}{\lambda}+\left[\frac{\pi}{\mu}-\frac{1}{\mu} \arctan (\mu \tan \gamma)\right]=\pi$,

$\forall k \in \mathbb{I N}, \lambda \geq 0, \mu \geq 0, \gamma=\pi-\beta$. 


\section{Propiedades del espectro de Fučik para el problema de Sturm - Liouville}

Aquí estudiaremos las propiedades de las curvas $F_{k}^{+}$y $F_{k}^{-}$del espectro de (3.1), cuyas ecuaciones se obtuvieron en la sección anterior. Para ello, introducimos la función

$$
g(z, \alpha)=\frac{1}{z}[\pi-\arctan (z \tan \alpha)], \quad z>0,0<\alpha<\frac{\pi}{2} .
$$

Esta función tiene las siguiente propiedades:

- $g$ es monótona decreciente en el primer argumento. Más aún, si $z$ crece en el intervalo $\langle 0,+\infty\rangle, g$ decrece de $+\infty$ a 0 , y si $z$ está en el intervalo $\left\langle z_{0},+\infty\right\rangle$, los valores de $g$ están en el rango de $g\left(z_{0}, \alpha\right)$ a 0 .

- Para $z>0$ (fijo), $g$ es monótona decreciente en $\alpha$. Más aún, si $\alpha$ crece de 0 a $\frac{\pi}{2}, g$ decrece de $\frac{\pi}{z}$ a $\frac{\pi}{2 z}$.

Empezamos con el estudio de las ramas impares $F_{2 k-1}^{+}$y $F_{2 k-1}^{-}$. De la proposición 3.1, tenemos que

$$
\begin{array}{ll}
F_{2 k-1}^{+}: & {\left[\frac{\pi}{\mu}-\frac{1}{\mu} \arctan (\mu \tan \alpha)\right]+\frac{(k-1) \pi}{\mu}+\frac{(k-1) \pi}{\lambda}+\left[\frac{\pi}{\lambda}-\frac{1}{\lambda} \arctan (\lambda \tan \gamma)\right]=\pi,} \\
F_{2 k-1}^{-}: & {\left[\frac{\pi}{\lambda}-\frac{1}{\lambda} \arctan (\lambda \tan \alpha)\right]+\frac{(k-1) \pi}{\mu}+\frac{(k-1) \pi}{\lambda}+\left[\frac{\pi}{\mu}-\frac{1}{\mu} \arctan (\mu \tan \gamma)\right]=\pi,}
\end{array}
$$

las cuales pueden escribirse como:

$$
\begin{array}{ll}
F_{2 k-1}^{+}: & g(\mu, \alpha)+\frac{(k-1) \pi}{\mu}+\frac{(k-1) \pi}{\lambda}+g(\lambda, \gamma)=\pi \\
F_{2 k-1}^{-}: & g(\lambda, \alpha)+\frac{(k-1) \pi}{\mu}+\frac{(k-1) \pi}{\lambda}+g(\mu, \gamma)=\pi
\end{array}
$$

Así tenemos los siguientes resultados:

Proposición 4.1 Para todo $k \in \mathbb{N}$, las ramas $F_{2 k-1}^{+}$y $F_{2 k-1}^{-}$son simétricas respecto a la diagonal en el primer cuadrante. Es decir, si $(\mu, \lambda) \in F_{2 k-1}^{+}$, entonces $(\lambda, \mu) \in F_{2 k-1}^{-} y$ viceversa.

Observación 4.1 Si $\alpha=\gamma$, entonces las ramas $F_{2 k-1}^{+}$y $F_{2 k-1}^{-}$coinciden. 
Proposición 4.2 La función $\mu=f(\lambda)$, dada por (4.1), decrece monótonamente.

Prueba.- Usando el hecho que $g(z, \alpha)$ y $\frac{\pi}{z}$ son funciones monótonas decrecientes en $z$, probaremos que $\lambda_{1}<\lambda_{2}$ implica que $\mu_{1}>\mu_{2}$. En efecto, tenemos las siguientes implicaciones:

$$
\begin{aligned}
\lambda_{1}<\lambda_{2} & \Longrightarrow g\left(\lambda_{1}, \gamma\right)+\frac{(k-1) \pi}{\lambda_{1}}>g\left(\lambda_{2}, \gamma\right)+\frac{(k-1) \pi}{\lambda_{2}} \\
& \Longrightarrow \pi-g\left(\lambda_{1}, \gamma\right)-\frac{(k-1) \pi}{\lambda_{1}}<\pi-g\left(\lambda_{2}, \gamma\right)-\frac{(k-1) \pi}{\lambda_{2}} \\
& \Longrightarrow g\left(\mu_{1}, \alpha\right)+\frac{(k-1) \pi}{\lambda_{1}}<g\left(\mu_{2}, \alpha\right)+\frac{(k-1) \pi}{\mu_{2}}
\end{aligned}
$$

De la última desigualdad se tiene que $\mu_{1}>\mu_{2}$.

Observación 4.2 Las ramas $F_{2 k-1}^{+}$y $F_{2 k-1}^{-}$son los gráficos de funciones monótonas decrecientes.

Proposición 4.3 Para la curva $F_{2 k-1}^{+}$existen la asíntota vertical $\lambda=\lambda_{2 k-1}^{+}$y la asíntota horizontal $\mu=\mu_{2 k-1}^{+}, \quad \forall k \in \mathbb{N}$.

Prueba.- Si $\mu \longrightarrow+\infty$, entonces $g(\mu, \alpha) \longrightarrow 0$ y $\frac{(k-1) \pi}{\mu} \longrightarrow 0$. De (4.1) tenemos que

$$
\frac{(k-1) \pi}{\lambda}+g(\lambda, \gamma) \longrightarrow \pi
$$

De esto, y usando el hecho que las funciones $\mu=f(\lambda)$ y $\frac{(k-1) \pi}{\lambda}+g(\lambda, \gamma)$ son monótonas, llegamos a que existe $\lambda_{2 k-1}^{+}$tal que $\lambda \longrightarrow \lambda_{2 k-1}^{+}$. El valor $\lambda_{2 k-1}^{+}$se halla de la ecuación

$$
\frac{(k-1) \pi}{\lambda}+g(\lambda, \gamma)=\pi
$$

0

$$
\pi(k-\lambda)=\arctan (\lambda \tan \gamma)
$$

Del mismo modo se prueba que existe una asíntota horizontal $\mu=\mu_{2 k-1}^{+}$.

El valor de $\mu=\mu_{2 k-1}^{+}$se puede hallar de

$$
\frac{(k-1) \pi}{\mu}+g(\mu, \alpha)=\pi,
$$

o

$$
\pi(k-\mu)=\arctan (\mu \tan \alpha) .
$$

Observación 4.3 Por la simetría de $F_{2 k-1}^{+}$y $F_{2 k-1}^{-}$respecto a la diagonal, existe una asíntota vertical $\lambda=\lambda_{2 k-1}^{-}$y una asíntota horizontal $\mu=\mu_{2 k-1}^{-}$para la curva $F_{2 k-1}^{-}, \forall k \in \mathbb{N}$. Los valores de $\lambda_{2 k-1}^{-}$y $\mu_{2 k-1}^{-}$se hallan de las ecuaciones

$$
\pi(k-\lambda)=\arctan (\lambda \tan \alpha) \quad y \quad \pi(k-\mu)=\arctan (\mu \tan \gamma),
$$

respectivamente. 
Observación 4.4 De las proposiciones 4.1 y 4.3 se tiene que $\lambda_{2 k-1}^{+}=\mu_{2 k-1}^{-}$y $\mu_{2 k-1}^{+}=$ $\lambda_{2 k-1}^{-}$.

Proposición 4.4 (Comparación de asíntotas) Las asintotas de $F_{2 k-1}^{+}$y $F_{2 k-1}^{-}$se relacionan de la siguiente forma:

Si $\alpha>\gamma$, entonces

$$
\lambda_{2 k-1}^{-}<\lambda_{2 k-1}^{+}, \quad \mu_{2 k-1}^{-}>\mu_{2 k-1}^{+} .
$$

Si $\alpha<\gamma$, entonces

$$
\lambda_{2 k-1}^{-}>\lambda_{2 k-1}^{+}, \quad \mu_{2 k-1}^{-}<\mu_{2 k-1}^{+} .
$$

Prueba.- Probaremos que $\alpha>\gamma$ implica $\lambda_{2 k-1}^{-}<\lambda_{2 k-1}^{+}$.

Observamos que $z_{1}=\lambda_{2 k-1}^{-}$y $z_{2}=\lambda_{2 k-1}^{+}$son soluciones de

$$
\pi(k-z)=\arctan (z \tan \alpha) \quad \text { y } \quad \pi(k-z)=\arctan (z \tan \gamma),
$$

respectivamente.

Como $\alpha>\gamma \quad$ y $\quad z>0$, tenemos que

$$
\tan \alpha>\tan \gamma \text { y } \arctan (z \tan \alpha)>\arctan (z \tan \gamma)
$$

y por tanto

$$
\pi\left(k-z_{1}\right)>\pi\left(k-z_{2}\right)
$$

de donde

$$
z_{1}=\lambda_{2 k-1}^{-}<z_{2}=\lambda_{2 k-1}^{+} \text {. }
$$

La relación $\mu_{2 k-1}^{-}>\mu_{2 k-1}^{+}$se obtiene del hecho que las ramas $F_{2 k-1}^{+}$y $F_{2 k-1}^{-}$son simétricas con respecto a la diagonal en el primer cuadrante.

Para el caso $\alpha<\gamma$, similarmente se prueba

$$
\lambda_{2 k-1}^{-}>\lambda_{2 k-1}^{+} \quad, \quad \mu_{2 k-1}^{-}<\mu_{2 k-1}^{+} .
$$

Ahora veamos las propiedades de las ramas pares $F_{2 k}^{+}$y $F_{2 k}^{-}$. Estas pueden escribirse como:

$$
\begin{aligned}
& F_{2 k}^{+}: \quad g(\mu, \alpha)+\frac{(k-1) \pi}{\mu}+\frac{k \pi}{\lambda}+g(\mu, \gamma)=\pi, \\
& F_{2 k}^{-}: \quad g(\lambda, \alpha)+\frac{k \pi}{\mu}+\frac{(k-1) \pi}{\lambda}+g(\lambda, \gamma)=\pi .
\end{aligned}
$$

Análogamente a los resultados para las ramas impares, tenemos los siguientes resultados:

Proposición 4.5 Las ramas $F_{2 k}^{+}$y $F_{2 k}^{-}$son simétricas respecto a la bisectriz del primer cuadrante.

Proposición 4.6 La función $\mu=f(\lambda)$ definida por la relación (4.4) decrece monótonamente. 
Observación 4.5 Las ramas $F_{2 k}^{+}$y $F_{2 k}^{-}$son los gráficos de funciones monótonas decrecientes. Proposición 4.7 Las curvas $F_{2 k}^{+}$tienen asintotas verticales $\lambda=\lambda_{2 k}^{+}$y asintotas horizontales $\mu=\mu_{2 k}^{+}, \forall k \in \mathbb{N}$.

Corolario 4.1 Para cualquier $k$, las curvas $F_{2 k}^{+}$tienen asíntota vertical común $\lambda_{2 k}^{+}=k$, cuando $\alpha$ y $\gamma$ difieren.

Observación 4.6 Por la simetría de las ramas $F_{2 k}^{+}$y $F_{2 k}^{-}$, existen asíntotas verticales y horizontales $\lambda=\lambda_{2 k}^{-}$y $\mu=\mu_{2 k}^{-}$para las curvas $F_{2 k}^{-}, \forall k \in \mathbb{I N}$.

El valor de la asíntota vertical $\lambda_{2 k}^{-}$puede hallarse de

$$
\pi((k+1)-\lambda)=\arctan (\lambda \tan \alpha)+\arctan (\lambda \tan \gamma),
$$

y la asintota horizontal es dada por

$$
\mu_{2 k}^{-}=k .
$$

Corolario 4.2 Para cualquier $k \in \mathbb{N}$, las ramas $F_{2 k}^{-}$tienen asíntota horizontal común $\mu_{2 k}^{-}=k$, cuando $\alpha$ y $\gamma$ difieren.

Observación 4.7 Por simetría (ver proposición 4.5) tenemos que $\lambda_{2 k}^{+}=\mu_{2 k}^{-}$y $\mu_{2 k}^{+}=\lambda_{2 k}^{-}$.

Proposición 4.8 (Comparación de asíntotas) Para las asíntotas de las ramas $F_{2 k}^{+}$y $F_{2 k}^{-}$ se cumplen las siguientes relaciones:

$$
\lambda_{2 k}^{-}>\lambda_{2 k}^{+}, \quad \mu_{2 k}^{-}<\mu_{2 k}^{+}, \quad \forall k \in \mathbb{I N} .
$$

Observación 4.8 A diferencia de las ramas impares, las ramas $F_{2 k}^{+}$y $F_{2 k}^{-}$no coinciden si $\alpha=\gamma$. En la siguiente sección veremos que $F_{2 k}^{+}$y $F_{2 k}^{-}$coinciden sólo si $\alpha=\gamma=\frac{\pi}{2}$.

\section{Casos específicos de problemas de frontera Sturm - Liouville}

5.1. Caso $\alpha=\frac{\pi}{4}, \beta=\frac{3 \pi}{4}$

Consideramos el problema

$$
\left\{\begin{array}{l}
w^{\prime \prime}=-\mu^{2} w^{+}+\lambda^{2} w^{-} \\
w(0) \cos \frac{\pi}{4}-w^{\prime}(0) \operatorname{sen} \frac{\pi}{4}=0 \\
w(\pi) \cos \frac{3 \pi}{4}-w^{\prime}(\pi) \operatorname{sen} \frac{3 \pi}{4}=0
\end{array}\right.
$$

que es equivalente al problema

$$
\left\{\begin{array}{l}
w^{\prime \prime}=-\mu^{2} w^{+}+\lambda^{2} w^{-} \\
w(0)=w^{\prime}(0) \\
w(\pi)=-w^{\prime}(\pi)
\end{array}\right.
$$


y en coordenadas polares:

$$
\left\{\begin{array}{l}
\varphi^{\prime}=\left\{\begin{array}{lll}
\mu^{2} \operatorname{sen}^{2} \varphi+\cos ^{2} \varphi, & \text { si } & \operatorname{sen} \varphi \geq 0 \\
\lambda^{2} \operatorname{sen}^{2} \varphi+\cos ^{2} \varphi, & \text { si } & \operatorname{sen} \varphi<0
\end{array}\right. \\
\varphi(0)=\frac{\pi}{4} \\
\varphi(\pi)=\frac{3 \pi}{4}
\end{array}\right.
$$

En este caso, por la proposición 3.1, obtenemos las siguientes expresiones para las ramas del espectro:

$$
\begin{aligned}
F_{0}^{+}: & \frac{\pi}{\mu}-2 \frac{\arctan \mu}{\mu}=\pi \\
F_{0}^{+}: & \frac{\pi}{\lambda}-2 \frac{\arctan \lambda}{\lambda}=\pi \\
F_{1}^{ \pm}: & \frac{\pi}{\mu}-\frac{\arctan \mu}{\mu}+\frac{\pi}{\lambda}-\frac{\arctan \lambda}{\lambda}=\pi \\
F_{2}^{+}: & \frac{2 \pi}{\mu}-2 \frac{\arctan \mu}{\mu}+\frac{\pi}{\lambda}=\pi \\
F_{2}^{-}: & \frac{2 \pi}{\lambda}-2 \frac{\arctan \lambda}{\lambda}+\frac{\pi}{\mu}=\pi \\
\vdots & \quad \frac{k \pi}{\mu}-\frac{\arctan \mu}{\mu}+\frac{k \pi}{\lambda}-\frac{\arctan \lambda}{\lambda}=\pi \\
F_{2 k-1}^{ \pm}: & \frac{(k+1) \pi}{\mu}-2 \frac{\arctan \mu}{\mu}+\frac{k \pi}{\lambda}=\pi \\
F_{2 k}^{+}: & \quad \frac{(k+1) \pi}{\lambda}-2 \frac{\arctan \lambda}{\lambda}+\frac{k \pi}{\mu}=\pi, \quad \forall k \in \mathbb{N} \\
F_{2 k}^{-}: & \quad \forall
\end{aligned}
$$

Cálculo de asíntotas:

Si $\mu \longrightarrow \infty$, la asíntota vertical es dada por $\lambda=\lambda_{k}^{ \pm}$y viceversa, si $\lambda \longrightarrow \infty$, la asíntota horizontal es dada por $\mu=\mu_{k}^{ \pm}$. Aquí presentamos algunas de tales asíntotas:

$$
\begin{array}{ll}
F_{0}^{+}: & \mu_{0}^{+} \approx 0,638322, \quad F_{0}^{-}: \lambda_{0}^{-} \approx 0,638322, \\
F_{1}^{ \pm}: & \mu_{1}^{ \pm} \approx 0,787637, \quad \lambda_{1}^{ \pm} \approx 0,787637, \\
F_{2}^{+}: & \mu_{2}^{+} \approx 1,39577, \quad \lambda_{2}^{+} \approx 1, \quad F_{2}^{-}: \mu_{2}^{-} \approx 1, \quad \lambda_{2}^{-} \approx 1,39577, \\
F_{3}^{ \pm}: & \mu_{3}^{ \pm} \approx 1,67161, \quad \lambda_{3}^{ \pm} \approx 1,67161, \text { etc } .
\end{array}
$$

Observación 5.1 En este caso las ramas del espectro no tienen asíntotas comunes. 


\subsection{Caso $\alpha=\frac{\pi}{2}, \beta=\frac{\pi}{2} \quad$ (El problema tipo Neuman)}

Aquí nuestro problema en estudio, se escribe

$$
\left\{\begin{array}{l}
w^{\prime \prime}=-\mu^{2} w^{+}+\lambda^{2} w^{-} \\
w(0) \cos \frac{\pi}{2}-w^{\prime}(0) \operatorname{sen} \frac{\pi}{2}=0 \\
w(\pi) \cos \frac{\pi}{2}-w^{\prime}(\pi) \operatorname{sen} \frac{\pi}{2}=0
\end{array}\right.
$$

esto es, el problema tipo Neuman

$$
\left\{\begin{array}{l}
w^{\prime \prime}=-\mu^{2} w^{+}+\lambda^{2} w^{-} \\
w^{\prime}(0)=w^{\prime}(\pi)=0
\end{array}\right.
$$

o, en términos de coordenadas polares

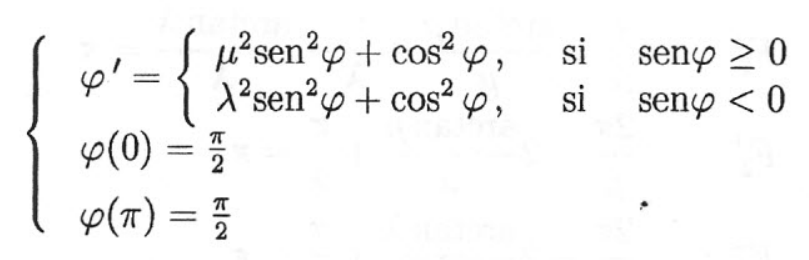

En este caso, por la proposición 3.1, obtenemos las siguientes expresiones para las ramas del espectro:

$$
\begin{aligned}
F_{0}^{+}: & \mu=0 \\
F_{0}^{+}: & \lambda=0 \\
F_{1}^{ \pm}: & \frac{1}{2 \mu}+\frac{1}{2 \lambda}=1 \\
F_{2}^{+}: & \frac{1}{\mu}+\frac{1}{\lambda}=1 \\
F_{2}^{-}: & \frac{1}{\mu}+\frac{1}{\lambda}=1 \\
\vdots & \\
F_{2 k-1}^{ \pm}: & \frac{2 k-1}{2 \mu}+\frac{2 k-1}{2 \lambda}=1 \\
F_{2 k}^{ \pm}: & \frac{k}{\mu}+\frac{k}{\lambda}=1 \\
\vdots & \frac{n}{2 \mu}+\frac{n}{2 \lambda}=1, \quad \forall n \in \mathbb{N} . \\
F_{n}^{ \pm}: &
\end{aligned}
$$

Ahora, calculamos sus asíntotas. Como en el caso anterior, si $\mu \longrightarrow \infty$ la asíntota vertical es dada por $\lambda=\lambda_{k}^{ \pm}$y viceversa, si $\lambda \longrightarrow \infty$, la asíntota horizontal es dada por $\mu=\mu_{k}^{ \pm}$. 
Así obtenemos las siguientes asíntotas:

$$
\begin{aligned}
F_{0}^{+}: & \mu=0, \quad F_{0}^{-}: \lambda=0 \\
F_{1}^{ \pm}: & \mu=0,5, \quad \lambda=0,5 \\
F_{2}^{ \pm}: & \mu=1, \quad \lambda=1 \\
F_{3}^{ \pm}: & \mu=1,5, \quad \lambda=1,5 \\
\vdots & \\
F_{n}^{ \pm}: & \mu=\frac{n}{2}, \quad \lambda=\frac{n}{2}, \quad \forall n \in \mathbb{N} .
\end{aligned}
$$

Observación 5.2 En este caso, las ramas del espectro tampoco tienen asíntotas comunes.

\section{Referencias}

[1] DANCER, E. N. -On the Dirichlet problem for weakly nonlinear elliptic partial equations. Proc. Roy. Soc. Edinburgh Sect. A 76, 1997, pág 283-300.

[2] FUČIK, S. and KUFNER, A. -Nonlinear Differential Eq́quations. Elsevier Scientific Publishing Company. The Netherlands, 1980.

[3] ROJAS, S. - Un ejemplo del espectro de Fučik. PESQUIMAT Revista de la Fac. CC. MM. de la UNMSM. Vol V. Nro. 2, Diciembre 2002, pág 55-63. 